\title{
Detecting Heel Strikes for Gait Analysis through Acceleration Flow
}

\author{
Yan Sun, Jonathon S. Hare*, Mark S. Nixon \\ School of Electronics and Computer Science, University of Southampton, Southampton, UK \\ *ish2@ecs.soton.ac.uk
}

\begin{abstract}
In some forms of gait analysis it is important to be able to capture when the heel strikes occur. In addition, in terms of video analysis of gait, it is important to be able to localise the heel where it strikes on the floor. In this paper, a new motion descriptor, acceleration flow, is introduced for detecting heel strikes. The key frame of heel strike can be determined by the quantity of acceleration flow within the Region of Interest (ROI), and positions of the strike can be found from the centre of rotation caused by radial acceleration. Our approach has been tested on a number of databases which were recorded indoors and outdoors with multiple views and walking directions for evaluating the detection rate under various environments. Experiments show the ability of our approach for both temporal detection and spatial positioning. The immunity of this new approach to three anticipated types of noises in real CCTV footage is also evaluated in our experiments. Our acceleration flow detector is shown to be less sensitive to Gaussian white noise, whilst being effective with images of low-resolution and without incomplete body position information when compared to other techniques.
\end{abstract}

\section{Introduction}

Gait analysis is the systematic study of human walking. It has been mainly applied in two fields: human identification [1] and medical consultation for diseases which affect walking [2]. Current gait analysis techniques can be classified as three types based on the sensor modality that is used to make measurements: physical-sensor based, depth image based and standard image based.

Physical-sensor based techniques are often used for clinical gait analysis; they measure the physical data, usually kinetic parameters, on the different parts of the walking body during gait cycles [3], [4]. The most popular wearable sensors are accelerometers and gyroscopes [5]. Milica and et al. [6] use accelerometer to measure the angle of leg segments and ankle. Rueterbories and et al. [7] use gyroscopes to capture the angular displacement, or Coriolis force since it is the response to a rotating particle, to discriminate gait events.

Depth, or RGBD image, based gait analysis techniques have expanded since the introduction and wide availability of PrimeSense and Kinect sensors. These measurements use the distance between the body parts and the sensor in depth images to analyse gait [8], [9]. Lu et al. [10] have built a gait database named ADSC-AWD based on Kinect data. O'Connor measure the acceleration of the body using Kinect.

Standard image-based gait recognition has been extensively studied. Most approaches are targeted at recognition of individual humans, using gait as the biometric signature. The general framework usually consists of background subtraction, feature extraction and classification [11]. The approaches can be classified into two catalogues: model-based and model-free. Model-based approaches have an intimate relationship with the human body and its motion. [12] presents an analytical gait model which extract the angle of thigh and lower leg rotation without parameter selection and [13] uses the statistical shape of the body for recognition. Model-free based approaches concentrate on the body shape or the motion of the entire gait process and thus could be used for the analysis of other moving shapes or mammals. Bobick and Davis [14] employ the motion-energy image and motion- history images of silhouette, Han and Bhanu [15] use the gaitenergy image for recognition. Model-based methods are view-invariant and scale-invariant but the computation cost is relatively high and the approaches can be very sensitive to image quality. Model-free approaches, an alternative to model-based approaches, are less sensitive to the image quality with lower computation cost though they are not intrinsically robust to variation in viewpoint and scale [11].

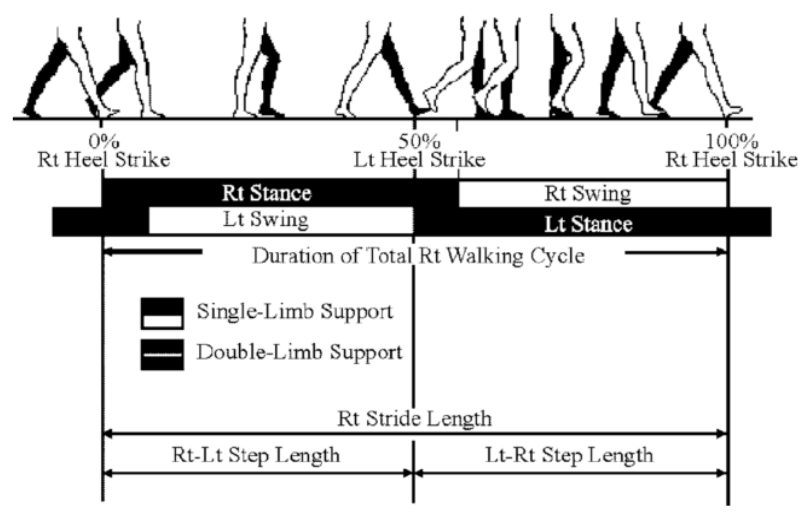

Fig. 1 The temporal components contained in a gait cycle and step and stride length during the cycle [27].

These gait analysis methods all rely on accurate gait events detection. The components of one gait cycle are shown in Fig. 1: a gait cycle is defined as the interval between two consecutive heel strikes of the same foot. A heel strike refers to the moment the heel first strikes the floor. Suppose one gait cycle starts from the heel strike of right foot, the right foot rotates on the heel to touch the floor ('stance phase') to support the body while the left foot is swinging forward ('swing phase') until the left heel strikes the floor. Then the roles of the two feet switch, the left foot remains flat on the floor whilst the right foot is swinging forward. When the right heel strikes the floor again, then a gait cycle is complete.

Hence the accurate and efficient detection of heel strikes is essential because it partitions a walking sequence into cycles composed of stance and swing phases [16]. In 
addition, the stride and step length can be derived from the stationary position of the heel at the moment of strike.

In this paper, we introduce a novel image-based technique to determine the moment and position of heel strikes by using a new motion descriptor: acceleration flow. Kinematic data has been commonly used in sensor-based approaches to indicate gait events: [7] and [17] use accelerometers; [18] and [19] use gyroscopes to measure the angular displacement on the subject to determine the gait phases.

In our contribution, we note that the change of velocity, which is acceleration, can also be determined by analysis of optical flow in image sequences. Standard optical flow algorithms focus on the displacement of brightness pattern, and do not consider high order motions or classification of different types of motion. To address this, we propose an algorithm to classify motion within image sequences and subsequently use the extracted acceleration field to detect heel strikes.

Previous standard image-based heel strike detection methods accumulate the image sequence of a gait cycle and determine the densest areas. They use corner [20] and silhouette [21] as the clues. Compared with these approaches, the temporal template of this new approach only requires three consecutive frames for processing and it also allows near real-time detection with only a single frame of delay.

The technique presented in this paper is based on the idea of differentiating motion types. Heel strikes incorporate radial acceleration on the foot while walking as they can be detected by the changes in angular velocity measured with gyroscopes [7]. In our approach, we first disambiguate acceleration and velocity from optical flow and then decompose the acceleration into tangential and radial components. Experimentation on multiple databases shows that our method reaches state of art accuracy on determining the timing of heel strikes, as well as demonstrating improved reliability on locating of each heel strike within the frame in which it occurs. An earlier version of this analysis showed how acceleration could be used to detect heel strikes [22] by applying a physical quantity to detect gait events based on a computer vision technique. There we classified acceleration and velocity from optical flow and decomposed acceleration along radial and tangential directions. We now extend the evaluation and explore ramifications of the new analysis. Beyond our previous work, this paper makes the following contributions:

- We evaluate the sensitivity of our approach to different imaging conditions via a wider range of datasets;

- We now evaluate different types of distortion: visual angle, lighting condition, Gaussian noise, occlusion and low resolution; and

- We compare the performance of our new operator with that of a previous technique and show performance improvement and capabilities.

The paper is organised as follows: Section 2 explains the details of acceleration flow and decomposition algorithms. Section 3 describes the methodology of applying acceleration flow to detect heel strikes. The experiments and analysis are presented in Section 4. Section 5 discusses the current heel strike detection techniques and the advantages and limitation of our algorithm. Finally, Section 6 concludes our work and explores potential future directions.

\section{Acceleration Estimation in Computer Images}

Previous research in motion analysis has usually used established techniques without considering the diversity of different types of motion [21], [23] and has typically focused on the determination of velocity flow. In fact, there are many different types of motion: in the simplest sense, there are objects that move with constant velocity and some that move with acceleration. If one looks more closely, it can be seen that the trajectory of an object is linked to its motion; an object moving at a constant velocity on a curved path is in fact undergoing acceleration as well. In kinetics, instantaneous acceleration is the derivative of instantaneous velocity with respect to time:

$$
\mathbf{a}=\lim _{\Delta t \rightarrow 0} \frac{\Delta \mathbf{v}}{\Delta t}
$$

To estimate the acceleration fields from image sequences, we formulate an approximation within three frames by considering the finite differences in the velocity fields between the current frame and the previous and following frames. The acceleration field $\mathbf{A}$ can be estimated by the temporal derivative of the optical flow between consecutive frames:

$$
\mathbf{A}(t)=\mathbf{V}(t+1)-(-\mathbf{V}(t))
$$

where $\mathbf{V}(t)$ and $\mathbf{V}(t+1)$ denote the motion fields from frame $t-1$ to $t$, and $t$ to $t+1$ separately. This formulation ensures reference to $t$ as the initial frame. In other words, the velocity fields in image sequences have the same starting frame by reversing the time axis from frame $t-1$ to $t$.

For this work, we use the state of the art optical flow technique, DeepFlow [26], to estimate the motion fields used to extract acceleration. Deepflow has excellent performance for large displacement estimation and non-rigid matching. We now decompose acceleration into radial and tangential components to determine their effects on motion respectively.

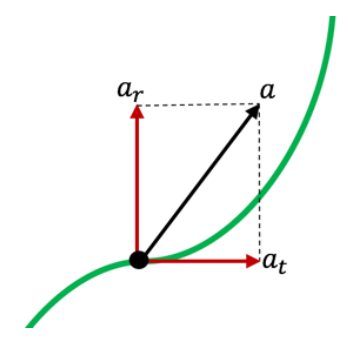

Fig. 2 The relationship between resultant, tangential and radial acceleration.

Decomposing acceleration into tangential and radial components is necessary for understanding the motion in image sequences. In kinematics, when a mass is moving along a curved path, the tangential acceleration changes the magnitude of velocity and the direction is in the tangent line of the trajectory (increasing or decreasing the speed). The radial acceleration (also called centripetal acceleration in circular motion) changes the trajectory and it points to the centre of the circular path (normal to the tangential direction), as shown in Fig. 2.

Furthermore, the trajectory of a mass can be considered as a straight line or a circumference of a circle if the period is short enough. Thus, we assume that the objects 
in the image sequence are moving along the same path within three consecutive frames and the trajectory is either a straight line or a circle. Then the estimated acceleration field can be decomposed into tangential and radial components since three points can determine one and only one circle or line. For determining the centre of rotation, we assume that the pixels tangential components. We shall now move to investigating the application of this approach in detecting heel strikes.

\section{Detecting Heel Strike for Gait Analysis by Acceleration}

Torsos move like connected pendula during walking,

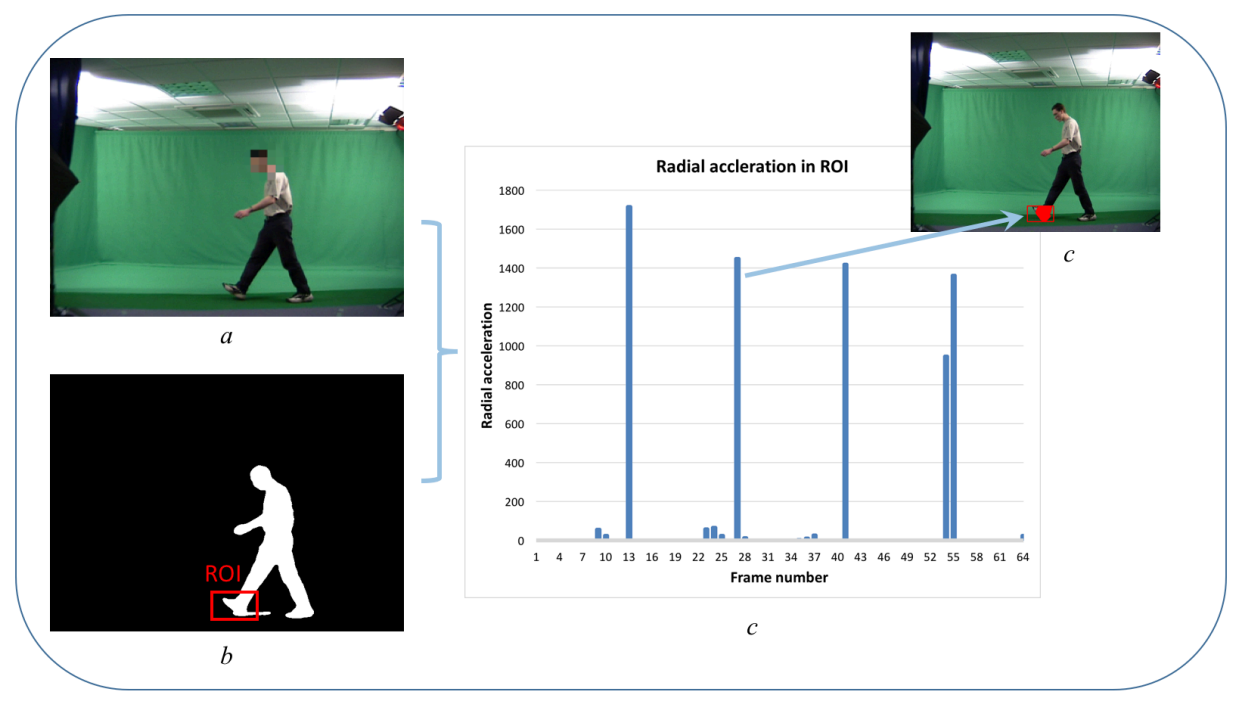

Fig. 3 An overview of key frame detection.

(a) The gait sequences.

(b) Extracted silhouettes and derived ROI.

(c) Detected radial acceleration in ROI.

which follow the circular motion rotate along the same circumference in any three consecutive frames because three points can decide one and only one circle.

Following the assumption, the radial component $\mathbf{a}_{\mathbf{r}}$ and tangential component $\mathbf{a}_{\mathbf{t}}$ can be estimated by their geometry relationship with the resultant acceleration $\mathbf{a}$ :

$$
\begin{aligned}
& \mathbf{a}_{\mathbf{r}}=\sin \theta * \mathbf{a} \\
& \mathbf{a}_{\mathbf{t}}=\cos \theta * \mathbf{a}
\end{aligned}
$$

where $\theta$ represents the angle between $\mathbf{a}$ and $\mathbf{a}_{\mathbf{t}}$ which equals to the angle between resultant acceleration $\mathbf{a}$ and velocity $\mathbf{v}$, as the velocity direction is along the tangent:

$$
\theta=\cos ^{-1} \frac{\mathbf{a} \cdot \mathbf{v}}{|\mathbf{a}||\mathbf{v}|}
$$

Then the rotation centre estimation of radial acceleration $\mathbf{a}_{\mathbf{r}}$ is similar with circular motion:

$$
\begin{gathered}
\mathbf{a}_{\mathbf{r}}=\mathbf{v}^{2} / r \\
r=\mathbf{v}^{2} /(\sin \theta * \mathbf{a})
\end{gathered}
$$

The centre $\left(o_{x}, o_{y}\right)$ can be derived by the coordinates of current processing pixel $\left(p_{x}, p_{y}\right)$ subtract the rotation radius:

$$
\begin{aligned}
& o_{x}=p_{x}-r \\
& o_{y}=p_{y}-r
\end{aligned}
$$

We now have the basis for detecting acceleration and its extension to a more generalized form in terms of radial and
[27] and [28] have successfully simulated pathological gait by using a liner inverted pendulum model. Penduli have a regular acceleration pattern, which implies that we can describe gait by the acceleration pattern of the image-based data. Fig. 4 shows the acceleration fields of the body during toe off (a), heel strike (b) and heel rise (c). They reveal that the legs and feet appear to have more acceleration or deceleration than the other parts of the body during different gait phases. Likewise, the forearms have acceleration since they are similar to swinging penduli. Therefore, the acceleration pattern of a walking body could be used to indicate the gait phases. In this work, we detect the heel strike by analysing the radial acceleration of the leading foot.

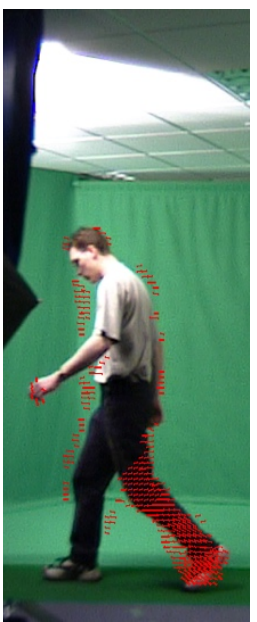

$a$

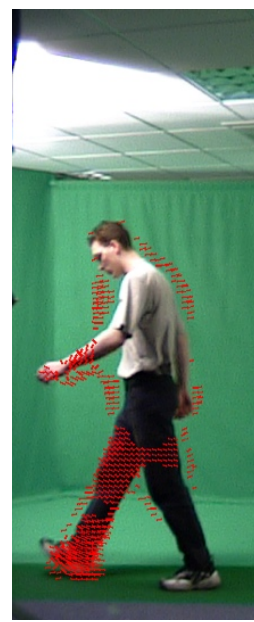

$b$

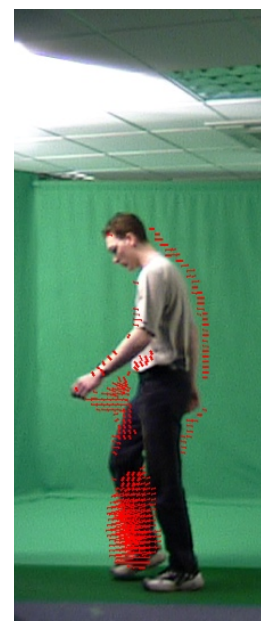

c
Fig. 4 The radial acceleration flow on a walking person. 
Table 1 Database information.

\begin{tabular}{lccccc}
\hline Databases & CASIA-A $\left(45^{\circ}\right)$ & CASIA-A $\left(90^{\circ}\right)$ & CASIA-B & SOTON & OU-ISIR \\
\hline Lighting Control & NO & NO & Yes & Yes & Yes \\
Camera Visual Angle $\left(^{\circ}\right)$ & 45 & 90 & 54 & 90 & $\sim 75$ \\
Number of subjects & 13 & 25 & 15 & 21 & 15 \\
Number of heel strike & 96 & 98 & 126 & 114 & 120 \\
Frame size & $240 \times 352$ & $240 \times 352$ & $240 \times 320$ & $576 \times 720$ & $480 \times 640$ \\
\hline
\end{tabular}

When the heel is approaching to strike, it changes from swinging forward to hit the ground and then the foot starts a circular motion which is centred at the heel. During this process, there is a large amount of radial acceleration flow on the front foot. Consequently, the key frame and location of heel strike can be estimated by detecting the radial acceleration on the foot. The framework of our heel strike detection system is illustrated in Fig. 3.

\subsection{Locating the frame of heel strike}

At the instant of heel strike, the heel hits the ground which forces the foot's velocity to cease in a short time. Therefore, the acceleration of the leading foot increases dramatically (rapid deceleration). Also, the striking foot sole's motion is approximately circular during the period between the heel striking on the ground to fully touching the ground, centred at the heel. Hence, most acceleration caused by heel strikes is radial in nature. The key frames where the heel strikes then can be located by the quantity of radial acceleration. Due to the similarity to penduli, as mentioned in acceleration pattern analysis on gait, the radial acceleration caused by a heel strike might be confused with that caused by other limbs. To reduce interference, we extract the region of interest (ROI) which is located on the leading foot according to a walking body model. The size of the ROI is $0.133 \mathrm{H} \times 0.177 \mathrm{H}$ where $\mathrm{H}$ represents the height, shown in Fig. 5.

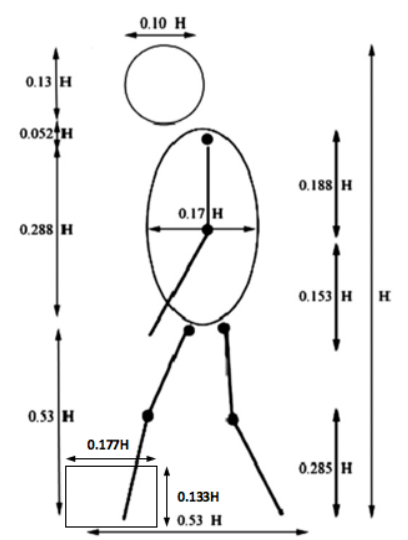

Fig. 5 Gait Proportion [33].

\subsection{Heel Position Estimation and verification}

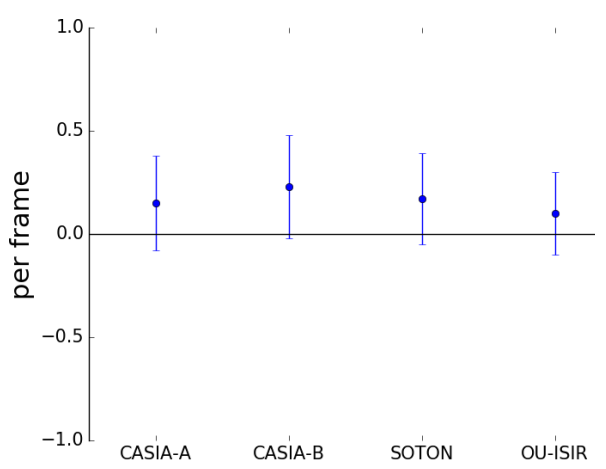

$a$

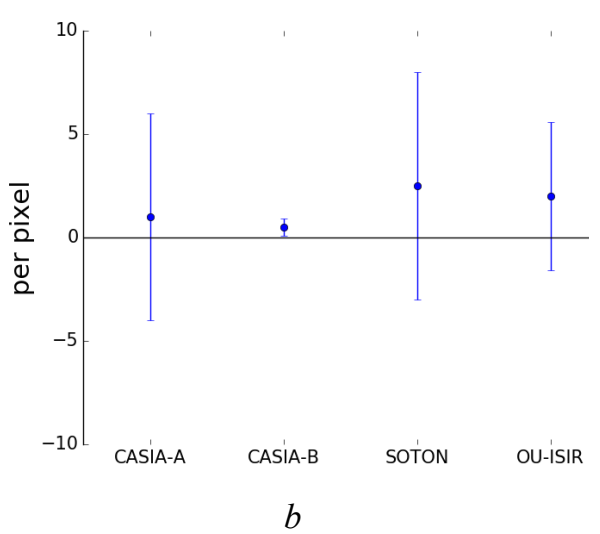

Fig. 6 Labelling error on different databases.

(a) Key frame, (b) Strike position.

If all the radial accelerations in the ROI are caused by the strike, their rotatin centres should all locate at the heel. A rotation centres accumulator map I is derived from the detected radial acceleration in the ROI. The weight of heel strike position $\left(i_{h}, j_{h}\right)$ is determined by the density map:

$$
\left[\begin{array}{l}
i_{h} \\
j_{h}
\end{array}\right]=\frac{1}{S} \sum_{i, j} \mathbf{I}(i, j) \times\left[\begin{array}{l}
i \\
j
\end{array}\right]
$$


where $\mathbf{I}(i, j)$ is the density at location $(i, j)$ and $S$ is the quantity of detected radial acceleration in the ROI.

\section{Experimental Results}

We evaluate our method on three benchmark databases: CASIA [29], [30], SOTON [31] and OU-ISIR [32]. The data used in this paper is collected with various controlled environments. We test around 100 heel strikes in each scenario and the test data incorporates multiple viewing angles and walking directions with gait sequences recorded indoors and outdoors, as described in Table 1. The ground truth of key frames and heel strike positions were manually labelled multiple times by different people. Fig. 6 shows the variance of manually labelled ground truth between different databases for key frames and strike positions. The variance in the key frame labelling is generally low and within one frame. Fig. 6 (b) shows greater variance on the SOTON dataset as it has the largest ROI compared with other databases.

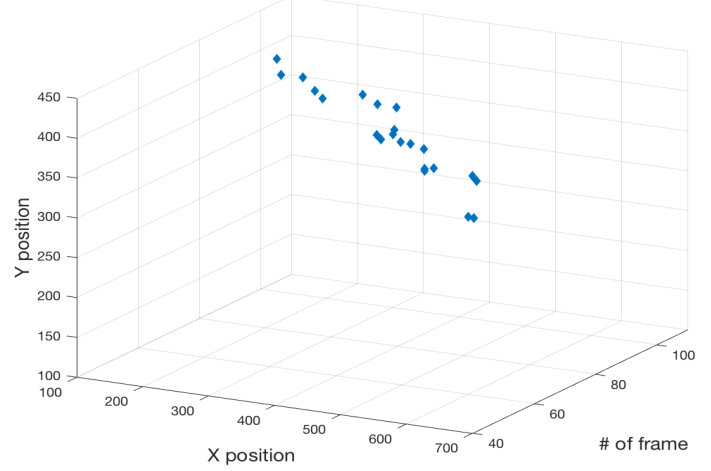

$a$

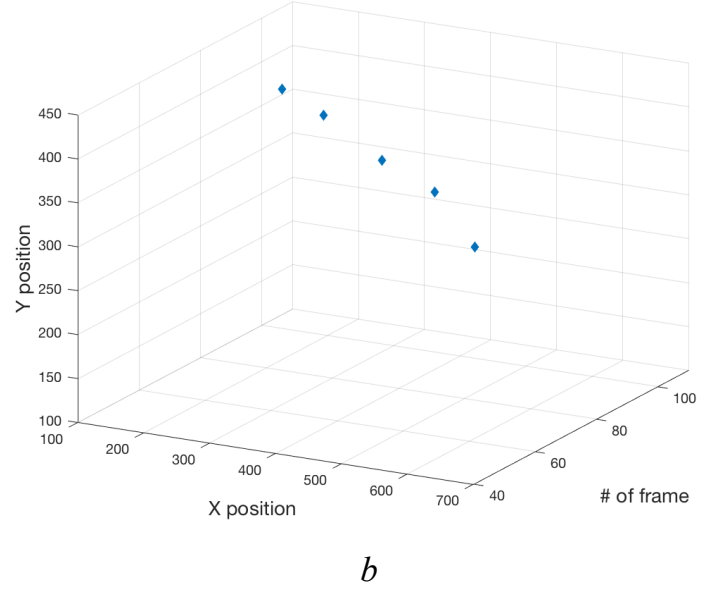

Fig. 7 Heel strike verification process.

(a) Candidates for heel strikes,

(b) Detected heel strikes (after filtering).

The acceleration decomposition algorithm is based on a subject moving perpendicular to the background so it is theoretically most effective in a direction perpendicular to the camera. Therefore, gait data imaged at multiple views has been used to evaluate the robustness of our approaches to other view angles. For further research and evaluation, the implementation code and heel strikes' ground truth are publicly available ${ }^{1}$.

\subsection{Key Frame Detection}

The moment of a heel strike is detected according to the quantity of radial acceleration in the ROI as described in Section 3. The histogram of radial acceleration within a walking sequence shows distinct suggestions for key frames, as shown previously in Fig.3. In the sequence, the radial acceleration appears regularly and noticeably, showing the periodicity of gait. The heel strikes occur at frame 13, 27, 41, and between frames 54 and 55 .

\subsection{Heel Strike Position Verification}

The ROI extracted according to gait proportions is not always perfectly located on the leading foot in the sequence because the shape of the human body changes during a gait cycle. Also, there is radial acceleration on the other body parts, for example the calf, since the limbs' motion is that of several joined pendulums [33]. The rotation centres of these erroneous radial accelerations also form invalid strike position candidates. To reduce the effect of this error, the detected key frames are used to filter the heel strike position candidates. When the heel strikes between two frames, the acceleration quantities are used as a weighting factor for deriving the positions. Fig. 7 (a) shows detected candidates of heel strike positions in each frame and (b) is the result after being filtered by key frames. The (expected) periodicity of gait is evident in the result.

Table 2 The accuracy of heel strike detection by corner and acceleration (values in \%).

\begin{tabular}{lll}
\hline Dataset & Corner & Acceleration \\
\hline & & \\
CASIA-A $\left(45^{\circ}\right)$ & 75.3 & 40.0 \\
CASIA-A $\left(90^{\circ}\right)$ & 82.9 & 81.2 \\
CASIA-B & 73.3 & 70.8 \\
SOTON & 86.9 & 89.7 \\
OU-ISIR & 65.8 & 73.1
\end{tabular}

Table 3 The precision of strike positions (values in $\%$ ).

\begin{tabular}{lll}
\hline Dataset & Corner & Acceleration \\
\hline & & \\
CASIA-A $\left(45^{\circ}\right)$ & 25.3 & 72.0 \\
CASIA-A $\left(90^{\circ}\right)$ & 46.6 & 88.9 \\
CASIA-B & 46.2 & 78.8 \\
SOTON & 20.2 & 85.2 \\
OU-ISIR & 12.5 & 82.1
\end{tabular}

\subsection{Detection Performance}

\footnotetext{
${ }^{1}$ https://github.com/YanSunSoton/HeelStrikeAcc.
} 
Bouchrika and Nixon proposed a method that accumulated corners within a gait cycle by the Harris corner detector to determine the positions of heel strikes.

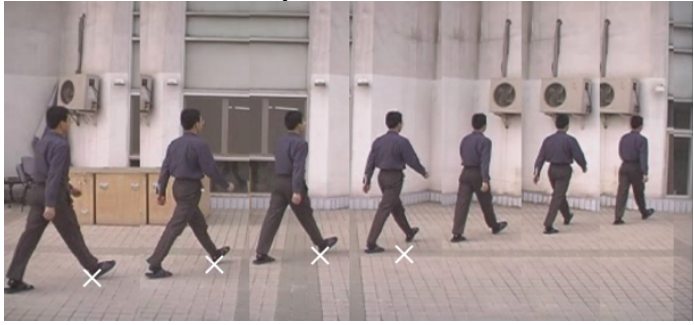

$a$

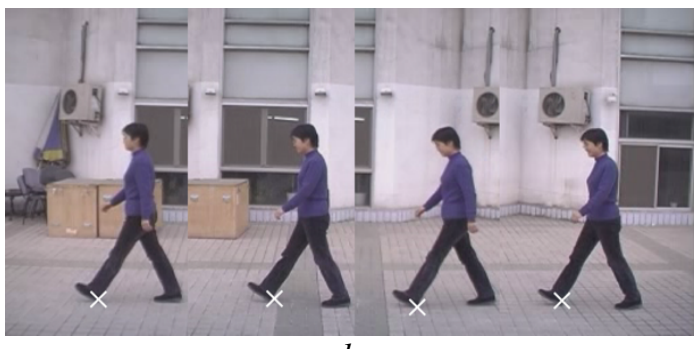

$b$

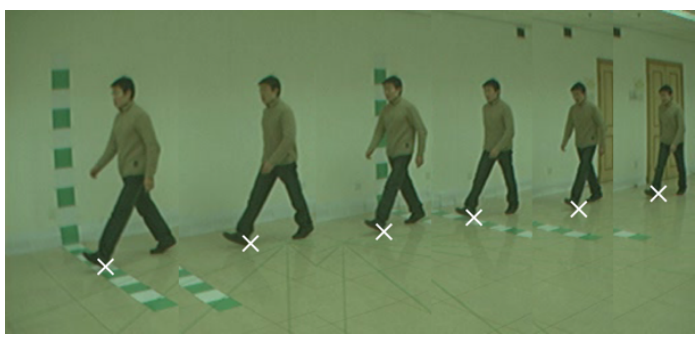

c

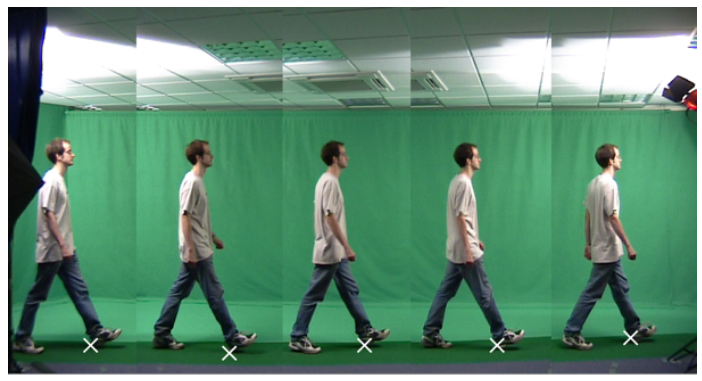

$d$

Fig. 8 Examples of detection results with various databases.
(a) CASISA-A (45 ),
(c) $C A S I A-B$,
(b) CASIA-A (90\%),
(d) SOTON.

Theoretically, there should be dense corners accumulated at the positions of heel since the heels stay at the strike positions for almost half gait cycle [34]. We compare our detection results against the corner detection method since there are few heel strike detection methods based on standard image sequences with implementation available. Tables 2 and 3 illustrate the comparative results of corner detection and acceleration detection. The results in these tables differ from the earlier results [22] because the background has been included to give a more realistic implementation scenario. The detection of the heel strike moments (the key frames) and positions are evaluated separately since they are determined individually and they describe different events in gait analysis. Table 2 shows the accuracy of key frame detection and Table 3 is the precision of heel strike positions. Since the corner detection does not return the key frames, an additional condition is applied which is that for a key frame to be successfully detected a corner position within \pm 30 pixels from the ground truth is considered as a true positive. This condition is actually quite generous and leads to an optimistic estimate of the frame for corner detection. For the radial acceleration detector, the criterion for a true positive key frame is whether the detection frame within \pm 1 frames from the ground truth. For heel positioning results of both methods, a distance within \pm 10 pixels (along both axes) from the ground truth is considered as an accurate location in Table 3.

In these results, the radial acceleration detector shows a much better ability for precise positioning for all the camera views. It is also able to detect key frames accurately when the camera view is nearly perpendicular to the subjects but the detection rate decreases with the increase of the angle between the camera and the walking subjects. Acceleration is more sensitive to the view angle since the magnitude of the detected acceleration is much smaller than the actual value if the walking trajectory is not perpendicular to the camera. The difference increases with the angle between them therefore a universal threshold becomes inappropriate. Fig. 8 shows samples of the detection results for different databases. In Fig. 8 (a), the acceleration detector failed to detect several strikes when the subject was walking away from the camera and the accuracy of localization also decreases.

\subsection{Robustness of Heel Strike Detection Approaches}

Since the performance of a system under adverse imaging conditions is important, we evaluate the robustness of our heel strike detection technique. Three different factors affecting image quality, that might reduce the detection rates, are applied to the original sequences: Gaussian zero-mean white noise, occlusion in the detection area, and reduced resolution. These factors reflect some of the difficulties anticipated when detecting heel strikes in real surveillance videos. Fig. 9 shows the examples of different types of noised gait images at different levels.

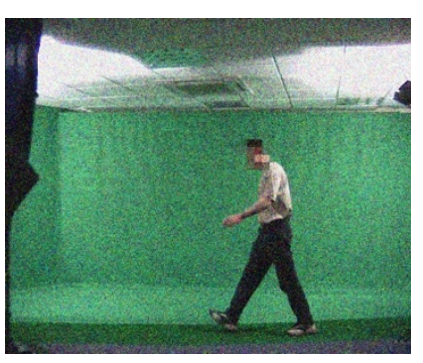

$a$

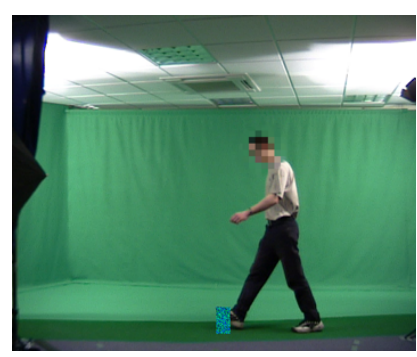

$b$
Fig. 9 Examples of added noise and occlusion.

(a) Gaussian white noise ( $\sigma=1.5 \%$ ),

(b) Occlusion (40\%).

Fig. 10 illustrates the results of testing the acceleration detector's immunity to these factors. Corner detection is also evaluated for comparison. The performance of the acceleration detector reduces slowly with the increase 
of Gaussian noise variance while corner detection is much more sensitive to the Gaussian noise, as shown in Fig. 10 (a).

The evaluation of immunity to occlusion investigates whether the gait information in real surveillance can be totally seen, or not. Occlusion was achieved by covering ROI from the toe to the heel increasingly with a random texture. The performance under occlusion decreases steadily, and our approach failed when ROI is covered more than $30 \%$ of the whole area. This is because most high-magnitude acceleration is located around the toe (the toe travels the greatest distance during heel strikes) but the toe is almost completely occluded when the occlusion in ROI is over $30 \%$. The detection by corners does not decrease significantly since the area that most corners are concentrated on the heel which has not been totally occluded yet. Acceleration does outperform the Corner method when occlusion is slight. If the occlusion were to start from heel to toe, acceleration flow detection could achieve much better immunity than the corner detector.

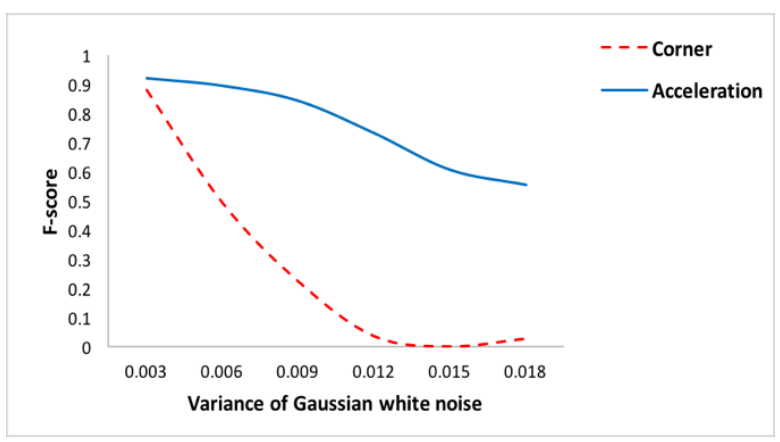

$a$
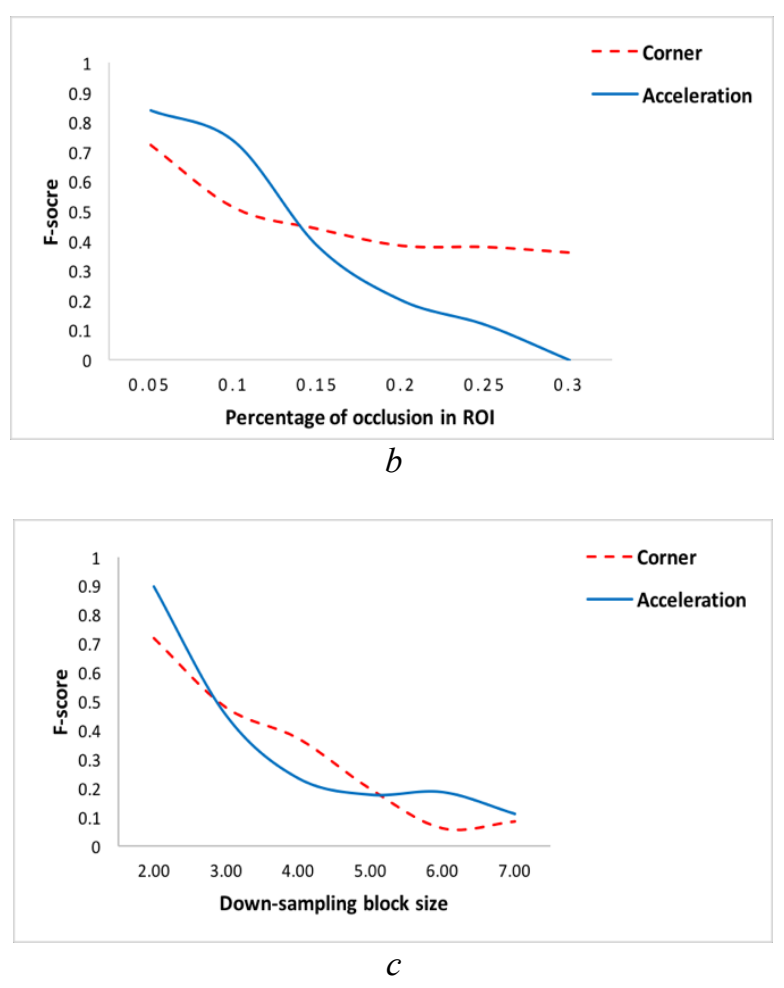

Fig. 10 Performance analysis of heel strike detection.

(a) Testing immunity to Gaussian white noise,

(b) Testing immunity to occlusion,

(c) Testing immunity to low resolution.
Resolution reduction investigates whether resolution of the subject is sufficient in surveillance footage. The original images are down-sampled and the detection rate of both approaches decreases to a low level when the new pixels are equivalent to $5 \times 5$ patches in the original image (in which the height of the subject is now around 70 pixels whereas it was 350 pixels originally). Acceleration and corner detectors show similar characteristics under this situation.

\section{Discussion}

In dynamics, the change of force causes acceleration, and acceleration changes motion. Consequently, acceleration is a distinctive cue to the change of motion. Some physicsbased gait analysis has used accelerometers and gyroscopes to detect the acceleration and angular velocity of the body parts to determine gait phases. We now apply this principle to standard image sequences to detect heel strikes. When the heel approaches strike, the foot has great amount of radial acceleration which is centred at the heel. Only the acceleration flow in the region around the leading foot is taken into account in our approach to reduce the effects of noise. Our experimental results show that acceleration is a more powerful way of estimating the positions of the strike than previous standard image-based technique. Also, our method overcomes the problem of detection in real-time as only three frames are needed for estimating acceleration flow. The evaluation of immunity to different types of noise suggests that acceleration is more robust to Gaussian noise than Bouchrika's approach.

On the other hand, the main limitation of acceleration is its sensitivity to the visual angle between camera and the subject. When the camera is orthogonal to the subject, acceleration performs best since the measurements and decomposition algorithms utilise a 2-D plane. The most realistic way of solving this problem at present is by applying the algorithm using a 3-D volume, for example using Kinect depth images, to replace the standard image sequence. However, the complexity of computation from this will be much higher than the existing technique.

Another weakness of this approach is it can only be applied in data with a clean background and the subjects in the images not overlap, which is similar to the most existing techniques. Currently background subtraction and silhouette extraction are still essential pre-processing for most standard image-based approaches. The results will be severely affected if the scene is too complex, for example in a crowded scene since the subjects' bodies overlap. Hence there is still refinement necessary to be able to apply these techniques in poor quality images, such as surveillance footage of the underground, or to videos recorded with adverse illumination.

\section{Conclusions and Ongoing Work}

This work uses acceleration to estimate the spatiotemporal position of heel strikes in standard image sequences for gait analysis. This is achieved by disambiguating acceleration and velocity from the displacement estimated by optical flow. In previous research on motion analysis in image sequences, only relative movement between consecutive frames has been considered. We have found that acceleration is much more distinctive than displacement or velocity in motion analysis so we derived an approach to extract acceleration from optical flow 
and used it to represent the change of motion. We have shown that it is possible to estimate acceleration from triplets of consecutive frames and that it can be decomposed into directional components, allowing selectivity of different types of motion. This allows greater depth in the understanding of motion sequences, and provides us with an approach to potentially extract more discriminative features.

We have shown that acceleration flow can be used for heel strike detection, since the foot undergoes a specific set of motion during heel strikes. Cunado et al. [33] proposed that the limbs appear to have pendulum-like motion in their gait model. In physics, the motion of a pendulum can be easily described by radial and tangential acceleration. Beyond that, acceleration has been widely used for recognition in physicalbased gait analyse techniques. Therefore, it will be propitious to investigate in the future whether the acceleration components in standard images can be used for recognition purposes.

Our experiments have investigated how camera viewpoint can affect performance, as radial and tangential components are derived based on a plane perpendicular to the subjects. The ability to detect heel strikes and estimate their positions using acceleration has been compared with one of the few existing techniques and the results show that whilst the new approach is more sensitive to the track of the walking subject relative to the camera. Our algorithm performs much better on heel strike positions estimation and appears more robust to Gaussian noise.

\section{Acknowledgments}

This manuscript would not have been possible without the help from Osaka University for providing us with access to the OU-ISIR database and Imed Bouchrika from the University of Souk Ahras who provided the implementation of his algorithm for heel strike detection by corners. We are also grateful to for The Institute of Automation, Chinese Academy of Sciences making their databases CASIA publicly available.

\section{References}

[1] M. S. Nixon, T. Tan, and R. Chellappa, Human identification based on gait. Springer, 2005.

[2] M. W. Whittle, Normal Ranges for Gait Parameters, Fourth Edi., vol. 3. Harrison Heidi, 2007.

[3] M. M. Skelly and H. J. Chizeck, "Real-time gait event detection for paraplegic FES walking," IEEE Trans. Neural Syst. Rehabil. Eng., vol. 9, no. 1, pp. 59-68, Mar. 2001.

[4] T. Chau and S. Rizvi, "Automatic stride interval extraction from long, highly variable and noisy gait timing signals," Hum. Mov. Sci., vol. 21, no. 4, pp. 495-514, Oct. 2002.

[5] P. B. Shull, W. Jirattigalachote, M. A. Hunt, M. R. Cutkosky, and S. L. Delp, "Quantified self and human movement: A review on the clinical impact of wearable sensing and feedback for gait analysis and intervention," Gait Posture, vol. 40, no. 1, pp. 11-19, May 2014.

[6] M. D. Djurić-Jovičić, N. S. Jovičić, and D. B. Popović, "Kinematics of gait: New method for angle estimation based on accelerometers," Sensors, vol. 11, no. 11, pp.
10571-10585, Nov. 2011.

[7] J. Rueterbories, E. G. Spaich, B. Larsen, and O. K. Andersen, "Methods for gait event detection and analysis in ambulatory systems," Med. Eng. Phys., vol. 32, no. 6, pp. $545-552,2010$.

[8] C. M. O'Connor, S. K. Thorpe, M. J. O'Malley, and C. L. Vaughan, "Automatic detection of gait events using kinematic data," Gait Posture, vol. 25, no. 3, pp. 469-474, 2007.

[9] E. Auvinet, F. Multon, C. E. Aubin, J. Meunier, and M. Raison, "Detection of gait cycles in treadmill walking using a Kinect," Gait Posture, vol. 41, no. 2, pp. 722-725, Feb. 2015.

[10] J. Lu, G. Wang, and P. Moulin, "Human identity and gender recognition from gait sequences with arbitrary walking directions," IEEE Trans. Inf. Forensics Secur., vol. 9, no. 1, pp. 51-61, Jan. 2014.

[11] J. Wang, M. She, S. Nahavandi, and A. Kouzani, "A Review of Vision-Based Gait Recognition Methods for Human Identification," in 2010 International Conference on Digital Image Computing: Techniques and Applications, 2010, pp. 320-327.

[12] C. Yam, M. Nixon, and J. Carter, "Gait recognition by walking and running: a model-based approach," Asian Conf. Comput. Vis., pp. 1-6, Jan 2002.

[13] L. Wang, T. Tan, W. Hu, and H. Ning, "Automatic gait recognition based on statistical shape analysis," IEEE Trans. Image Process., vol. 12, no. 9, pp. 1120-1131, Sep. 2003.

[14] A. F. Bobick and J. W. Davis, "The recognition of human movement using temporal templates," IEEE Trans. Pattern Anal. Mach. Intell., vol. 23, no. 3, pp. 257-267, Mar. 2001.

[15] J. Han and B. Bhanu, "Individual recognition using gait energy image," IEEE Trans. Pattern Anal. Mach. Intell., vol. 28, no. 2, pp. 316-322, Feb. 2006.

[16] J. A. Zeni, J. G. Richards, and J. S. Higginson, "Two simple methods for determining gait events during treadmill and overground walking using kinematic data," Gait Posture, vol. 27, no. 4, pp. 710-714, May 2008.

[17] A. Hreljac and R. N. Marshall, "Algorithms to determine event timing during normal walking using kinematic data," J. Biomech., vol. 33, no. 6, pp. 783-786, 2000.

[18] I. P. I. Pappas, M. R. Popovic, T. Keller, V. Dietz, and M. Morari, "A reliable gait phase detection system," IEEE Trans. Neural Syst. Rehabil. Eng., vol. 9, no. 2, pp. 113125, Jun. 2001

[19] S. Bamberg, a. Y. Benbasat, D. M. Scarborough, D. E. Krebs, and J. a. Paradiso, "Gait Analysis Using a ShoeIntegrated Wireless Sensor System," IEEE Trans. Inf. Technol. Biomed., vol. 12, no. 4, pp. 413-423, Jul. 2008.

[20] I. Bouchrika and M. S. Nixon, "Model-Based Feature Extraction for Gait Analysis and Recognition," Comput. vision/computer Graph. Collab. Tech., pp. 150-160, 2007.

[21] S. U. Jung and M. S. Nixon, "Heel strike detection based on human walking movement for surveillance analysis," Pattern Recognit. Lett., vol. 34, no. 8, pp. 895-902, Jun. 2013.

[22] Y. Sun, J. S. Hare, and M. S. Nixon, "Detecting Acceleration for Gait and Crime Scene Analysis," in 

and Prevention, 2016.

[23] S. Baker, D. Scharstein, J. P. Lewis, S. Roth, M. J. Black, and R. Szeliski, "A database and evaluation methodology for optical flow," Int. J. Comput. Vis., vol. 92, no. 1, pp. 131, Mar. 2011.

[24] D. Sun, S. Roth, and M. J. Black, "A quantitative analysis of current practices in optical flow estimation and the principles behind them," Int. J. Comput. Vis., vol. 106, no. 2, pp. 115-137, 2014.

[25] D. Fortun, P. Bouthemy, and C. Kervrann, "Optical flow modeling and computation: A survey," Comput. Vis. Image Underst., vol. 134, pp. 1-21, 2015.

[26] P. Weinzaepfel, J. Revaud, Z. Harchaoui, and C. Schmid, "DeepFlow: Large displacement optical flow with deep matching," in Proceedings of the IEEE International Conference on Computer Vision, 2013, pp. 1385-1392.

[27] T. Komura, A. Nagano, H. Leung, and Y. Shinagawa, "Simulating pathological gait using the enhanced linear inverted pendulum model," IEEE Trans. Biomed. Eng., vol. 52, no. 9, pp. 1502-1513, 2005.

[28] S. Kajita, O. Matsumoto, and M. Saigo, "Real-time 3D walking pattern generation for a biped robot with telescopic legs," Proc. 2001 ICRA. IEEE Int. Conf. Robot. Autom., vol. 3, pp. 2299-2306, 2001.

[29] S. Yu, T. Tan, K. Huang, K. Jia, and X. Wu, "A study on gait-based gender classification," IEEE Trans. Image Process., vol. 18, no. 8, pp. 1905-1910, Aug. 2009.

[30] L. Wang, H. Ning, T. Tan, and W. Hu, "Fusion of Static and Dynamic Body Biometrics for Gait Recognition," IEEE Trans. Circuits Syst. Video Technol., vol. 14, no. 2, pp. 149-158, Feb. 2004.

[31] J. Shutler, M. Grant, M. S. Nixon, and J. N. Carter, "On a Large Sequence-Based Human Gait Database," Proc. Fourth Int. Conf. Recent Adv. Soft Comput., pp. 66-72, 2002.

[32] H. Iwama, M. Okumura, Y. Makihara, and Y. Yagi, "The OU-ISIR gait database comprising the large population dataset and performance evaluation of gait recognition," IEEE Trans. Inf. Forensics Secur., vol. 7, no. 5, pp. 15111521, Oct. 2012.

[33] D. Cunado, M. S. Nixon, and J. N. Carter, "Automatic extraction and description of human gait models for recognition purposes," Comput. Vis. Image Underst., vol. 90, no. 1, pp. 1-41, 2003.

[34] I. Bouchrika and M. S. Nixon, "Gait-based pedestrian detection for automated surveillance," Proc. Int. Conf. Comput. Vis. Syst., 2007. 